\title{
Three-dimensional ultrasound and HDlive in tubal serous cystadeno- fibroma: a case report and literature review
}

\author{
Mihaela Grigore ${ }^{1}$, Razvan Popovici ${ }^{1}$, Cristina Furnica ${ }^{2}$, Anda Pristavu ${ }^{1}$, Alexandru Hamod ${ }^{3}$, \\ Dumitru Gafitanu ${ }^{1}$
}

${ }^{1}$ Department of Obstetrics and Gynecology, ${ }^{2}$ Department of Anatomy, ${ }^{3}$ Student,University of Medicine and Pharmacy

"Grigore T. Popa" Iasi, Romania

\begin{abstract}
Cystadenofibromas of the fallopian tubes are very rare benign tumors and very few cases have been reported in the literature worldwide. Usually, the tumor is asymptomatic, and for almost all cases reported, the tumors were incidentally discovered during surgery for other genital pathology. We report the case of a 30-year-old woman with a serous cystadenofibroma of the fallopian tube, presenting with chronic abdominal pain and secondary infertility. The diagnosis of tubal tumor was formulated before surgery and confirmed during laparoscopic surgery. Both 3D ultrasound and HDlive were useful tools for the diagnosis. The ultrasound diagnosis was helpful in planning appropriate surgical management.
\end{abstract}

Keywords: fallopian tube; serous cystadenofibroma; 3D ultrasound; HDlive; minimally invasive surgery.

\section{Introduction}

Cystadenofibromas of the fallopian tubes are rare benign tumors and very few cases have been reported in the literature worldwide [1]. Usually, the tumor is asymptomatic, and for almost all cases reported, the tumors were incidentally discovered during surgery for other genital pathology. We report the case of a 30-year-old woman with a serous cystadenofibroma of the fallopian tube, presenting with chronic abdominal pain and secondary infertility. The diagnosis of a tubal tumor was formulated before surgery with two-dimensional and three-dimensional ultrasound(US) and confirmed during laparoscopic surgery. Both 2D and 3D US were helpful in planning appropriate surgical management.

Received 30.01.2017 Accepted 21.03.2017

Med Ultrason

2017, Vol. 19, No 4, 444-446

Corresponding author: Mihaela Grigore, $\mathrm{MD}, \mathrm{PhD}$

University of Medicine and Pharmacy

"Grigore T. Popa" Iasi, Romania

Department of Obstetrics and Gynecology

16 Universitatii street, 700115 Iasi, Romania

Phone: 0040744438024

E-mail: Mihaela.grigore@edr.ro

\section{Case report}

A 30-year-old woman, gravida 1, parous 1, presented for a gynecologic examination for chronic pelvic pain and secondary infertility. The endovaginal ultrasound revealed a septate uterus and a complex structure between the uterus and left ovary. The structure was $4 \mathrm{~cm}$ in diameter, with regular wall and fine papillae on the internal surface (fig 1a). The 2D Doppler examination showed no signal inside the papillae. The three-dimensional (3D) sonography with HDlive software (Voluson E8, 3D endovaginal probe $4-8 \mathrm{~Hz}$, GE Healthcare) of the tumor revealed a fine, regular aspect of the vegetation inside the tumor (fig 1b, 1c). The tumoral markers (CA 125, CA199) were in normal range. Because of the complex aspect and lack of connections between the ovary and the tumor, a fallopian tube tumor was suspected. Moreover, because of the 3D HDlive aspect and the normal value of the tumoral markers, we suspected a benign tumor. After discussing the management options with the patient, we performed a laparoscopic cystectomy. During the surgery, we confirmed the tubal tumor diagnosis and performed a cystectomy. The tumor was attached to the serosal surface of the tube (fig 1d). The histology results confirmed 


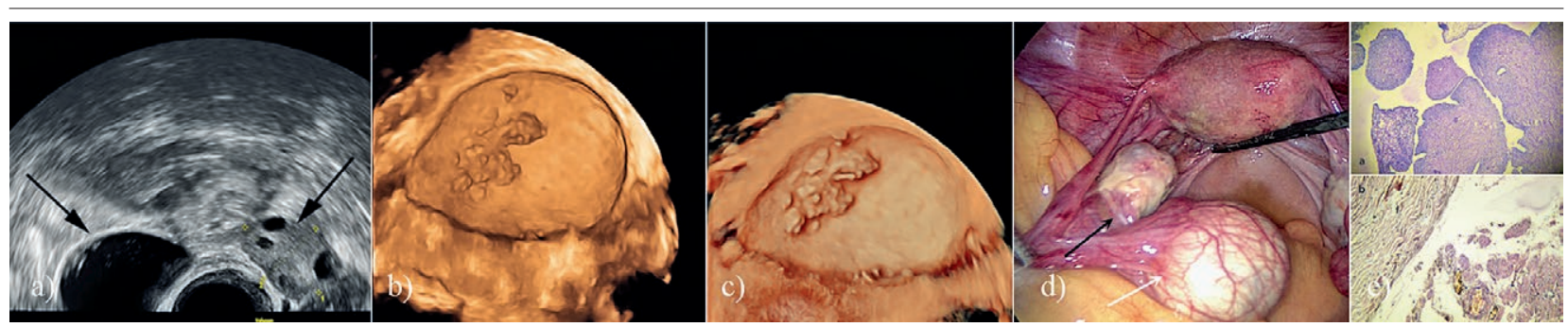

Fig 1. a) Endovaginal 2Dultrasonography of the tumour (black arrow - ovary, white arrow - tumour with papillae inside); b) 3D ultrasound of the tumor - tumor pappilae are seen inside of the tumour; c) HDlive three-dimensional ultrasound of the tumor the pappilae are clearly seen; d) Laparoscopic image of the tumor (black arrow - left ovary, white arrow - fallopian tumour); d) histopathological examination - cystadenofibroma (up) and cyst wall and fallopian tube (down) (H\&E staining).

serous cystadenofibroma of the fallopian tube (fig 1e). The patient recovered uneventfully and was discharged three days postoperatively. Two months after the surgery, she became spontaneously pregnant. The pregnancy was in the left part of the uterus.

\section{Discussions}

Cystadenofibromas of the fallopian tube are very rare benign tumors with low malignancy potential [2]. In 1909, Iwanov reported the first case in the literature [3]. The origin of this tumor is not clear. Because this tumor is rare, it is considered to be an embryologic remnant rather than a proliferating neoplastic process. This theory is supported by Gurbuz who demonstrated that topography, histology and immunohistology suggested that the tumor he reported was an embryologic remnant originating from the müllerian duct [4].

The tumor is usually a round, solitary mass that has developed either intraluminally or has attached to the serosal surface or fimbriated part of the fallopian tube. The internal and/or external surface can be fimbriated or papillary. Two histological components are present, a connective tissue stroma without nuclear pleomorphism or mitosis and papillary structures on the surface of the tubal structures lined by epithelial cells [1].

Upon conducting a Medline search with "fallopian tube" and "cystadenofibroma" as search terms, we found seven cases in the English literature [2,4-8]. Most cases reported in the literature were asymptomatic, and the majority were incidental findings at the time of an operation for another gynecologic and non-gynecologic disorder. In all cases, in which the tumor was discovered incidentally, a salpingectomy or adnexectomy were performed. For only one case, in which the tumor was discovered during an in vitro fertilization evaluation, a cystectomy was performed [7].

The diagnosis of cystadenofibroma could be difficult because macroscopically and ultrasonographically these tumors could appear malignant, resulting in radical surgery [2]. In our case, anticipating the diagnosis before surgery was helpful in deciding the type of surgery to perform. We believe that with a careful ultrasound examination, combining 2D Doppler ultrasound and 3D ultrasound, the diagnosis could be at least suspected. A helpful tool could be HDlive technology, which uses an adjustable light source, allowing the operator the possibility to create lighting and shadowing effects, thereby increasing depth perception. This technology is useful for a better assessment of the interior wall of the cysts [9].

In our case, the patient's symptoms disappeared after surgery. One month after surgery without any other treatment, she became pregnant. Of particular interest was that she probably became pregnant with the help of the left fallopian tube because the gestational sac was in the left hemiuterus and the patient's uterus was septate. Because of the good prognosis and low malignant potential, it is important to establish this diagnosis before planning surgery in young patients who desire fertility. However, in patients over 50 years of age, presenting with any ovarian or fallopian tube tumor, there is no need for a conservative approach [2].

In conclusion although the cases presented to date were incidentally discovered, our case supports the idea that with thorough clinical and ultrasound examination, the diagnosis could be anticipated before surgery. The correct diagnosis is important especially in young patients who desire fertility and ultrasound examination is particularly important before planning the surgery.

\section{References}

1. Tavassoli FA, Devilee P (eds). WHO Classification of Tumors: Pathology and genetics of tumours of the breast and female genital organs. IARC Press, 2003.

2. de Silva TS, Patil A, Lawrence RN. Acute presentation of a benign cystadenofibroma of the fallopian tube: a case report. J Med Case Rep 2010;4:181. 
3. Iwanow WM. Cystadenofibroma papilliferum tubae Fallopie. Zentalbl Gynaekol 1909;33:745.

4. Gurbuz Y, Ozkara SK. Immunohistochemical profile of serous papillary cystadenofibroma of the fallopian tube: a clue of paramesonephritic origin. Appl Immunohistochem Mol Morphol 2003;11:153-155.

5. Silverman AY, Artenian B, Sabin M. Serous cystadenofibroma of the fallopian tube: a case report. Am J Obstet Gynecol 1978;130:593-595.

6. Valerdiz Casasola S, Pardo Mindan J. Cystadenofibroma of fallopian tube. Appl Pathol 1989;7:256-259.
7. Sills ES, Kaplan CR, Perloe M, Tucker MJ. Laparoscopic approach to an uncommon adnexal neoplasm associated with infertility: serous cystadenofibroma of the fallopian tube. J Am Assoc Gynecol Laparosc 2003; 10:545-547.

8. Erra S, Costamagna D. Serous cystadenofibroma of the Fallopian tube: case report and literature review. G Chir 2012;33:31-33.

9. Grigore M. HDlive imaging of a serous ovarian borderline ovarian tumor. Ultrasound Obstet Gynecol 2013;41:598599. 